\title{
Characterization and bioactivity study of nanohydroxyapatite on superhydrophilic vertically aligned carbon nanotubes using optical techniques
}

\author{
Joao Anderson Ferreira Irineu ${ }^{\mathrm{a}}$, Teresa Cristina Marsi ${ }^{\mathrm{a}}$, Tiago Goncalves Santos ${ }^{\mathrm{b}}$, Ana Maria \\ Espirito Santo $^{c}$, Joao Lucas Rangel ${ }^{\mathrm{b}}$, Ursula A. Mengui ${ }^{4}$, Airton Abrahao Martin ${ }^{\mathrm{b}}$, Evaldo Jose \\ Corat $^{\mathrm{d}}$, Fernanda Roberta Marciano ${ }^{\mathrm{a}}$ and Anderson Oliveira Lobo ${ }^{\mathrm{a}^{*}}$ \\ ${ }^{a}$ Laboratorio de Nanotecnologia Biomedica (NanoBio), Universidade do Vale do Paraiba, Sao Jose \\ dos Campos, SP, Brazil. \\ ${ }^{\mathrm{b}}$ Laboratorio de Espectroscopia Vibracional Biomedica (LEVB), Universidade do Vale do Paraiba, \\ Sao Jose dos Campos, SP, Brazil. \\ ${ }^{c}$ Departamento de Ciencias Exatas e da Terra, Instituto, Universidade Federal de Sao Paulo, \\ Diadema, SP, Brasil \\ ${ }^{\mathrm{d}}$ Laboratorio Associado de Sensores e Materiais (LAS), Instituto Nacional de Pesquisas Espaciais, \\ Sao Jose dos Campos, SP, Brazil.
}

\begin{abstract}
Vertically-aligned multi-walled carbon nanotubes (VACNT) is of particular interest in regenerative medicine. Templateinduced hydroxyapatite (HA) has broad prospects in applied fields of bone regenerative medicine. Thus, it becomes very attractive a combination these two excellent materials to bone tissue engineering applications. In this study the HA/VACNT nanocomposites were used as scaffolds to Human osteoblast cells culture. Superhydrophilic VACNT films were obtained by CVD method and funcionalized by oxygen plasma. The fabrication of HA/VACNT nanocomposites was performed with a direct electrodeposition of the thin HA films on the VACNT films. The bioactivity and biomineralization in vitro process of superhydrophilic HA/VACNT nanocomposites were investigated using simulated body fluid (SBF) and optical techniques. The characterization of of HA/VACNT nanocomposites was performed before and after soaking 21 days in SBF and compared to superydrophilic VACNT films. Fourier transform infrared spectroscopy, micro X-ray fluorescence spectrometer by energy-dispersive and X-ray difractogram were employed to investigate the structural and chemical properties. The morphology was investigated by FEG-SEM analysis. After 21 days was identified that others biological apatites were formed only on HA/VACNT nanocomposites. Optical techniques showing a powerful tool to characterizated and investigated the bioactivity in vitro process. These findings were very atractive to application of this new nanocomposite to bone tissue regeneration.
\end{abstract}

Keywords: nanohydroxyapatite; carbon nanotubes; vertically aligned; biomineralization; bioactivity and nanocomposites

*Corresponding author: Anderson de Oliveira Lobo (A. O. Lobo). Email: loboao@yahoo.com. Tel: +551239471100.

Colloidal Nanocrystals for Biomedical Applications VII, edited by Wolfgang J. Parak, Kenji Yamamoto, Marek Osinski, Proc. of SPIE Vol. 8232, 82321K · (C) 2012 SPIE · CCC code: 1605-7422/12/\$18 · doi: 10.1117/12.907093 


\section{INTRODUCTION}

Hydroxyapatite (HA, Ca10(PO4)6(OH)2) is a form of calcium phosphate that bears close chemical resemblance with the mineral component of bones and teeth tissues [1]. It promotes tissue adhesion and bone growth by instinctively forming a biologically active bonelike apatite layer [2]. Thus, HA is classified as biocompatible and bioactive materials. Also is used for biological applications, such as dental or skeletal implants and bone repair scaffolds. Template-induced HA has broad prospects in the applied fields of regenerative medicine and bone repair. In order to create novel nanobiomaterials that mimic bone, it is essential to develop strategies to obtain a crystalline structure that is favorable to increase the osteointegration due to bioactivity properties. Studies on biomineralization mechanism have been aimed to develop a detailed understanding of interfacial interaction associated to biomineralization and template-directed crystallization [3]. Among other nanobiomaterials, vertically aligned carbon nanotubes (VACNT) undertake a great role for the study of tissue regeneration. The VACNTs have diameters around 10-100 nm, similar to the physical dimensions of extracellular matriz proteins. Hence, CNTs are particularly promising for tissue regeneration $[4,5,6]$.

We have shown a new biomimetic mineralization routine employing the HA/VACNT nanocomposites as highly stable template materials. The biomineralization was obtained after VACNT soaking in a simulated body fluid (SBF) know to favor the calcification process. The HA/VACNT calcification were unambiguously characterized and discussed by X-ray diffraction (XRD), Raman spectroscopy, Fourier transform infrared spectrophotometry (FTIR-ATR), $\mu$ EDX analyses and field emission gun scanning electron microscopy (FEG-SEM).

\section{MATERIALS AND METHODS}

The VACNTs were produced using a microwave plasma chamber $(2,45 \mathrm{GHz})$. Titanium squares were used as substrates. For VACNT syntheses were used titanium square substrates (covered by thin Fe layer $(7 \mathrm{~nm})$. The Fe layers were pretreated during $5 \mathrm{~min}$ in plasma of $\mathrm{N} 2 / \mathrm{H} 2(10 / 90 \mathrm{sccm})$ to promote nanoclusters formation. After this, was inserted $\mathrm{CH} 4$ $(14 \mathrm{sccm})$ to VACNT growth (2 minutes). The reactor was kept at a pressure of 30 Torr and temperature of $7600 \mathrm{C}$ during the whole process. More details about VACNT syntheses are shown in reference [7].

Superhydrophilic VACNT were obtained after a simple functionalization of the VACNT tips using a pulsed-direct current plasma reactor with an oxygen flow. More details in references [8, 9].

The electrodeposition of the HA crystals on the superhydrophilic VACNT films was performed using 0.042 molL-1 $\mathrm{Ca}(\mathrm{NO} 3) 2.4 \mathrm{H} 2 \mathrm{O}+0.025$ molL-1 (NH4).2HPO4 electrolytes (pH=4.7). Superhydrophilic VACNT films were used as working electrode. A platinum coil wire served as auxiliary electrode and an $\mathrm{Ag} / \mathrm{AgCl}$ electrode was used as reference electrode. The HA films were produced applying a constant potential of $-2.0 \mathrm{~V}$ during $30 \mathrm{~min}$ and the solution temperature was maintained at $70 \mathrm{oC}$. More details about electrodeposition of HA on the superhydrophilic VACNT films are shown in reference [10].

The SBF (5x) solution was used for in vitro bioactivity study. They were prepared by Barrere method [11]. For this the dissolution of $\mathrm{NaCl}, \mathrm{KCl}, \mathrm{K} 2 \mathrm{HPO} 4, \mathrm{CaCl} 2 \cdot 2 \mathrm{H} 2 \mathrm{O}, \mathrm{MgCl} 2 \cdot 6 \mathrm{H} 2 \mathrm{O}, \mathrm{NaHCO} 3, \mathrm{Na} 2 \mathrm{SO} 4$ and $(\mathrm{Na} 2 \mathrm{O}) \cdot \mathrm{SiO} 2$ all of analytical purity in distilled and deionized water. The $\mathrm{pH}$ of all solutions was adjusted to 7.25 at $37{ }^{\circ} \mathrm{C}$ with $1 \mathrm{~N} \mathrm{HCl}$ and tris(hidroxymetil)aminomethane. The solutions were kept in closed polyethylene containers. Superhydrophilic HA/VACNT composites were placed in a polyethylene recipient and were immersed in $15 \mathrm{ml}$ of SBF. All the substrates in their respective recipient were put in an incubator at $37^{\circ} \mathrm{C}$ for 21 days. After this incubation period, the implants were immersed in distilled and deionized water and finally dried at room temperature [9].

The structural analysis of biological apatites on superhydrophilic VACNT were performed at room temperature by X-ray diffractometry using X-Pert Philips with $\mathrm{CuK} \alpha$ radiation $(\lambda=0.154056 \mathrm{~nm})$ from 10 to $70^{\circ}$ in $2 \theta$ with the following condition voltage of $40 \mathrm{kV}$ and current of $30 \mathrm{~mA}$, step size of $0.02^{\circ}$ and a counting time of $2 \mathrm{~s}$ per step. Surface chemical compositions of the biological apatites were investigated by Fourier transform infrared attenuated total reflection spectroscopy (FT-IR ATR: Spectrum Spotlight-400, Perkin Elmer).

Semi-quantitative elemental analyses of calcium $(\mathrm{Ca})$ and phosphorus $(\mathrm{P})$ were carried out by a micro $\mathrm{X}$-ray fluorescence spectrometer by energy-dispersive ( $\mu$-EDX 1300, Shimadzu, Kyoto, Japan), equipped with a rhodium X-ray tube and a $\mathrm{Si}(\mathrm{Li})$ detector cooled by liquid nitrogen (N2). The equipment was coupled to a computer system for data processing. The energy range of scans was from 0.0 to $40.0 \mathrm{eV}$. The voltage in the tube was set at $15 \mathrm{kV}$, with automatic current adjustment. The analyses of $\mathrm{Ca}$ and $\mathrm{P}$ characteristic emissions were taken longitudinally on the sample surfaces, with incident beam diameter of $50 \mu \mathrm{m}$. The stepping mapping was taken using $40 \times 30$ points with a step of $20 \mu \mathrm{m}$ along the biological apatites formed on superhydrophilic VACNT films. Consequently, the analysis was performed in a line 100 $\mu \mathrm{m}$ long and $50 \mu \mathrm{m}$ thick. The scans were performed with a count rate of $10 \mathrm{~s}$ per point (live time) and a dead time of 
25\%. The equipment was adjusted using a certified commercial reagent of stoichiometric hydroxyapatite (Aldrich, synthetic Ca10(PO4)6(OH)2, grade $99.999 \%$, lot 10818HA) as reference. The measurements were collected using the fundamental parameters of characteristic X-ray emission of the elements $\mathrm{Ca}$ and $\mathrm{P}$. The elements $\mathrm{O}$ and $\mathrm{H}$ were used as chemical balance. The reference was also used as a point in the intensity curve calibration. The energy calibration was performed using internal standards for light elements [12].

\section{RESULTS AND DISCUSSIONS}

Figure 1 shows the morphological and structural VACNT analyses before and after exposure to the oxygen plasma. Figure 1a shows a SEM image of the high density of the VACNT film as-grown on Fe catalyst. VACNTs presented a length of $6-8 \mu \mathrm{m}$. Figure $1 \mathrm{~b}$ show a TEM image of typical internal bamboo-like structures of the VACNT. Figure 1c shows a SEM image after the oxygen plasma etching and figure 1d shows structural changes by TEM. No significant morphological or structural changes (only the tips) could be observed on converting to superhydrophilic VACNT films.
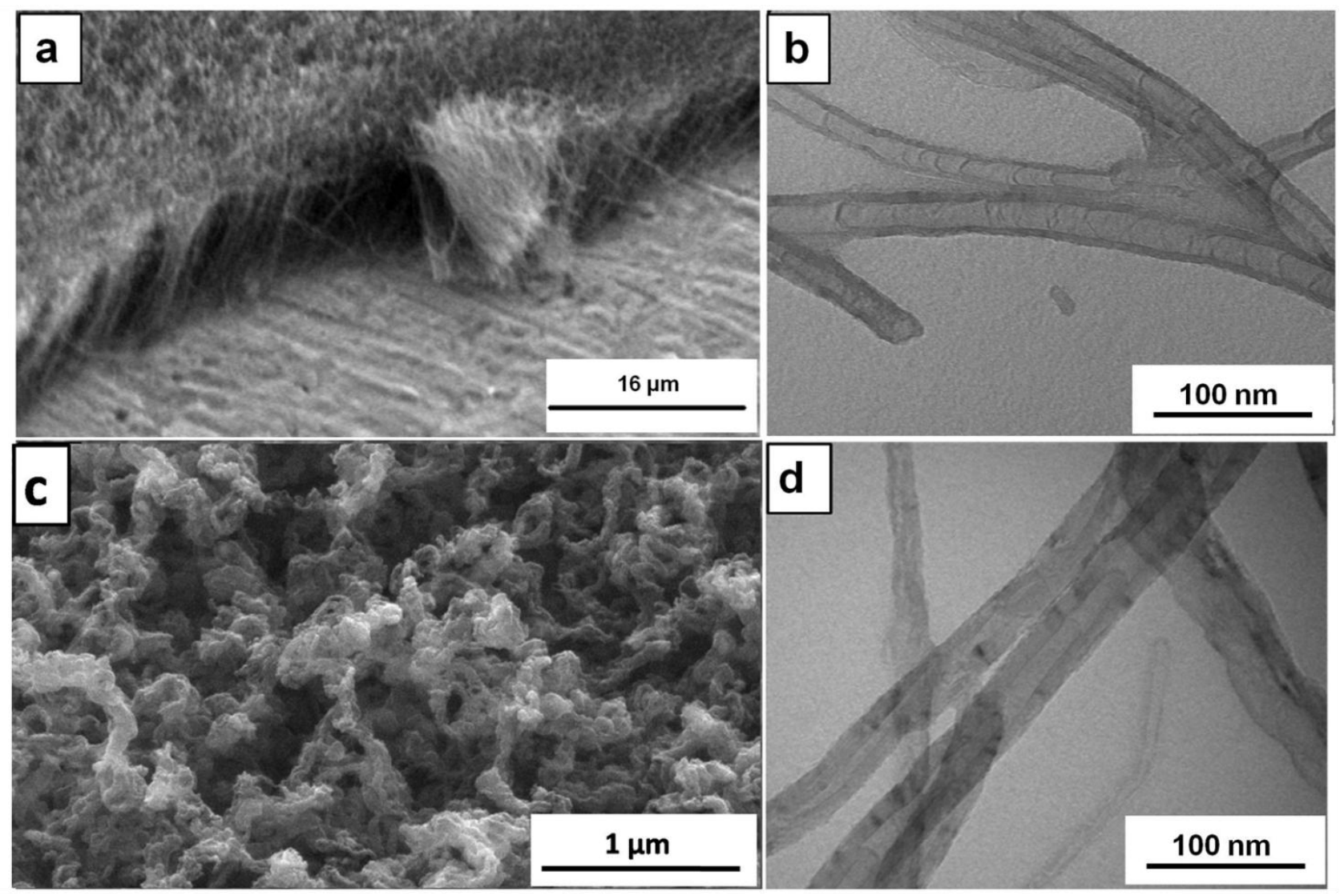

Figure 1. SEM images, of the as-grown (a) and (c) superhydrophilic VACNT films obtained by Fe catalysis. TEM images of bamboo-like structure of (b) as-grown and (d) superhydrophilic VACNT after the oxygen plasma etching.

The superhydrophilicity of the VACNT films obtained after the oxygen plasma treatment (data shown in reference [9]) was a requirement for obtaining the HA crystals (more details about HA electrodeposition are shown in elsewhere [10]), and consequently the excellent bioactivity presented here. This is a new method to obtain biomineralized HA/VACNTs nanocomposites.

Figure 2 show the SEM images of the HA/VACNT films before (a) and after 21 times of SBF incubation (b). The left picture is a top view, the central picture is the top view at a higher magnification and, the right picture is a tilted view to show the cross section details. In general, HA/VACNT nanocomposites are bioactive. Commonly, globular apatites, 
including biological ones, shows up when SBF is used in this biomimetic approach. The SEM images of Fig. $2 b$ show the massive growth of the apatite crystallites clusters with a globular-like shape on the HA/VACNTs nanocomposites. This cross section (Fig. 2b) view show the biological apatites formed on and between the HA/VACNT nanocomposites. It is very illustrative to define a completely densification among the HA/VACNT. From these findings, clearly we have shown the exceptional bioactivity of HA/VACNTs nanocomposites. We have shown an increase of the apatite layer deposited (thickness between 1.5-2.0 $\mu \mathrm{m}$ ).
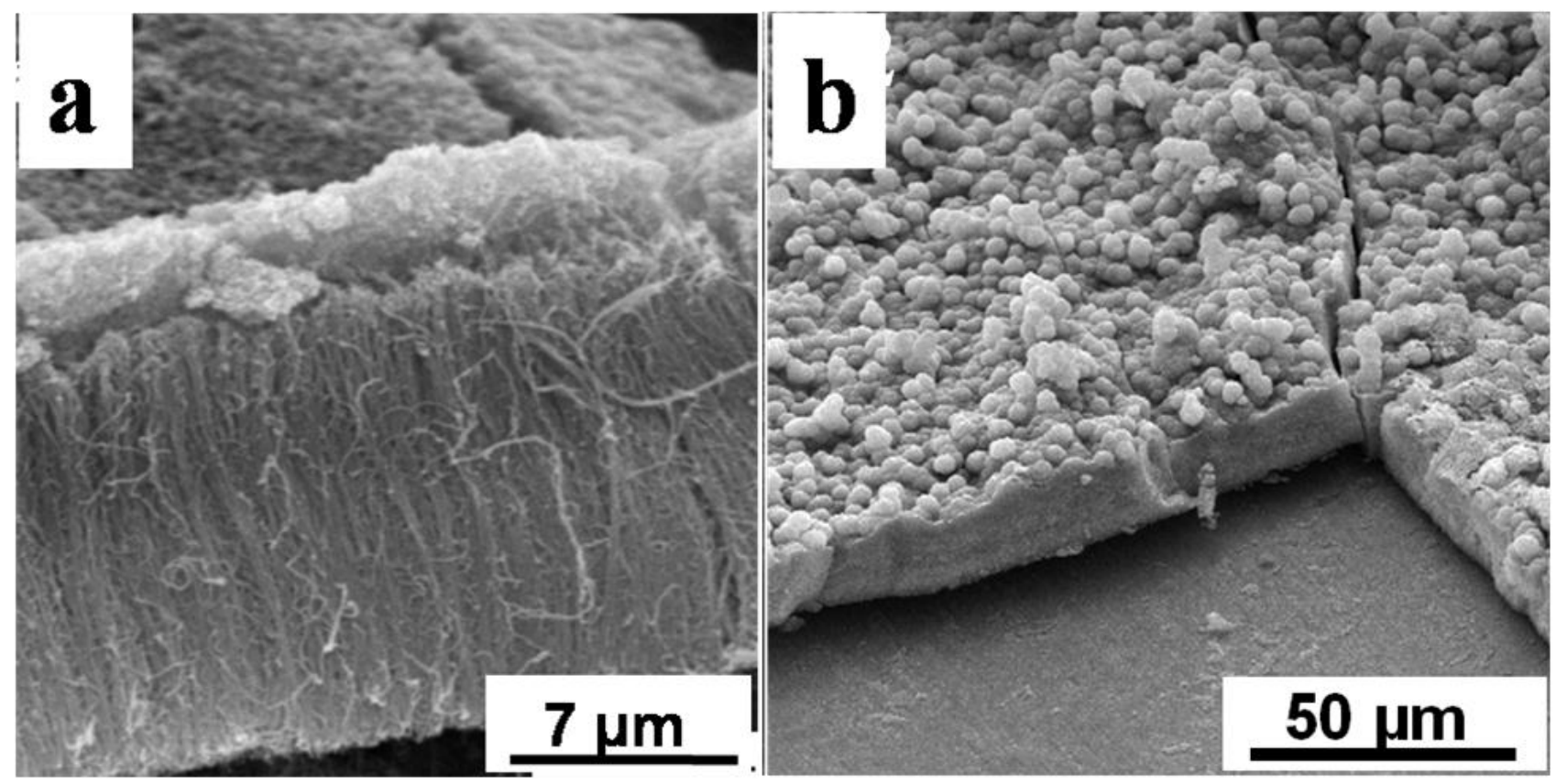

Figure 2. (a) SEM image of the cross section of HA film on top of the VACNT. (b) FEG-SEM images shown the bioactivity of HA/VACNTs nanocomposites after 21 days soaked in SBF. The cross section show the densification of HA/VACNTs nanocomposites.

Figure 3A shows the FT-IR ATR analysis. The multiplets located around $1000 \mathrm{~cm}-1$ are attributed to phosphate modes. The split bands, mainly at 1030 and $1090 \mathrm{~cm}-1$, seem to correlate with the formation of a well-crystallized apatite. A more detailed analysis indicates the presence of a carbonated component, where carbonate ions are substituting in A and B sites of the apatite structure [13] (corresponding to phosphate and hydroxyl ions, respectively). Carbonate bands have been detected at 879, 1415 and $1455 \mathrm{~cm}-1$. Molecular and adsorbed water bands are also seen at $1640 \mathrm{~cm}-1$ (Figure 3a). These results are very illustrative and conclusive to determine the bioactivity of HA/VACNT nanocomposites.

Fig. 3B shows the XRD diffraction patterns of the superhydrophilic VACNT, HA/VACNT nanocomposites produced by electrodeposition technique, and nanocomposites after the imersed in SBF after 21 days. For comparison was used the peaks of hydroxyapatite indexed in JCPDS (09-0432).

Figure 3B presents reflections in $25.9^{\circ}(002)$, and three peaks in the region around of $31.6^{\circ}$ (triplet ) characteristic of the planes (211), (112) and (300), and $34.0^{\circ}$ (200). This reflections was compared with the crystallographic identification pattern of HA published by JCPDS card number 09-0432, and were used to assign hexagonal indices and determined lattice parameters $a=0.9388 \mathrm{~nm}$ and $\mathrm{c}=0.6864 \mathrm{~nm}$. The presence of a strong peaks around of 31.5 and $37.04^{\circ}$ can be associated with the presence of calcite. And as has been discussed the formation of $\mathrm{HA}$ rightly $\mathrm{Ca} / \mathrm{P}$ greater than the theoretical value implies the formation of calcite during the production process. What this system may favor the formation of calcite [14]. It may be pointed out in this diffracttion, the peak intensity of calcite due to the biomineralization in vitro process [15]. The XRD results were confirmed by FT-IR, according to Tasis et al. the simultaneous occurrence of peaks at 711,870 and $1420 \mathrm{~cm}-1$ indicate the presence of calcite [14]. The temperature control in $37^{\circ} \mathrm{C}$ of the samples immersed in SBF can produce sharper peaks than those under ambient conditions [16], 
and the strong intensity the peaks indicated a very crystalline calcite. According to Tasis et al. [15] of the six phases of calcium carbonate (amorphous calcium carbonate, calcium carbonate hexahydrate, calcium carbonate monohydrate, vaterite, aragonite and calcite), calcite is the most thermodynamically stable at room temperature which favors their formation, even a little above these conditions. Other studies involving immersion of carbon nanotubes with some type of functionalization at soaked in SBF also found phase of calcite on the surface of the nanotubes after a certain time of immersion [14-16].

XRD

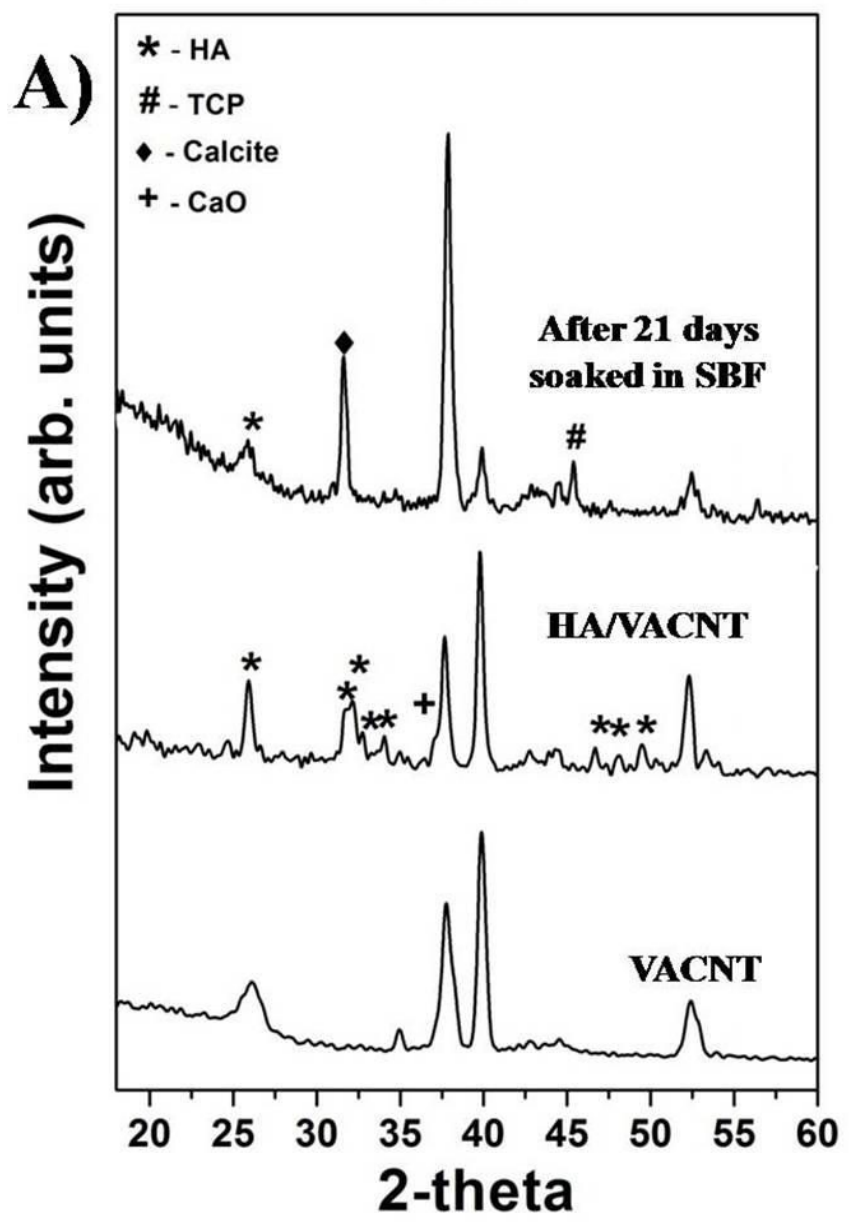

FTIR

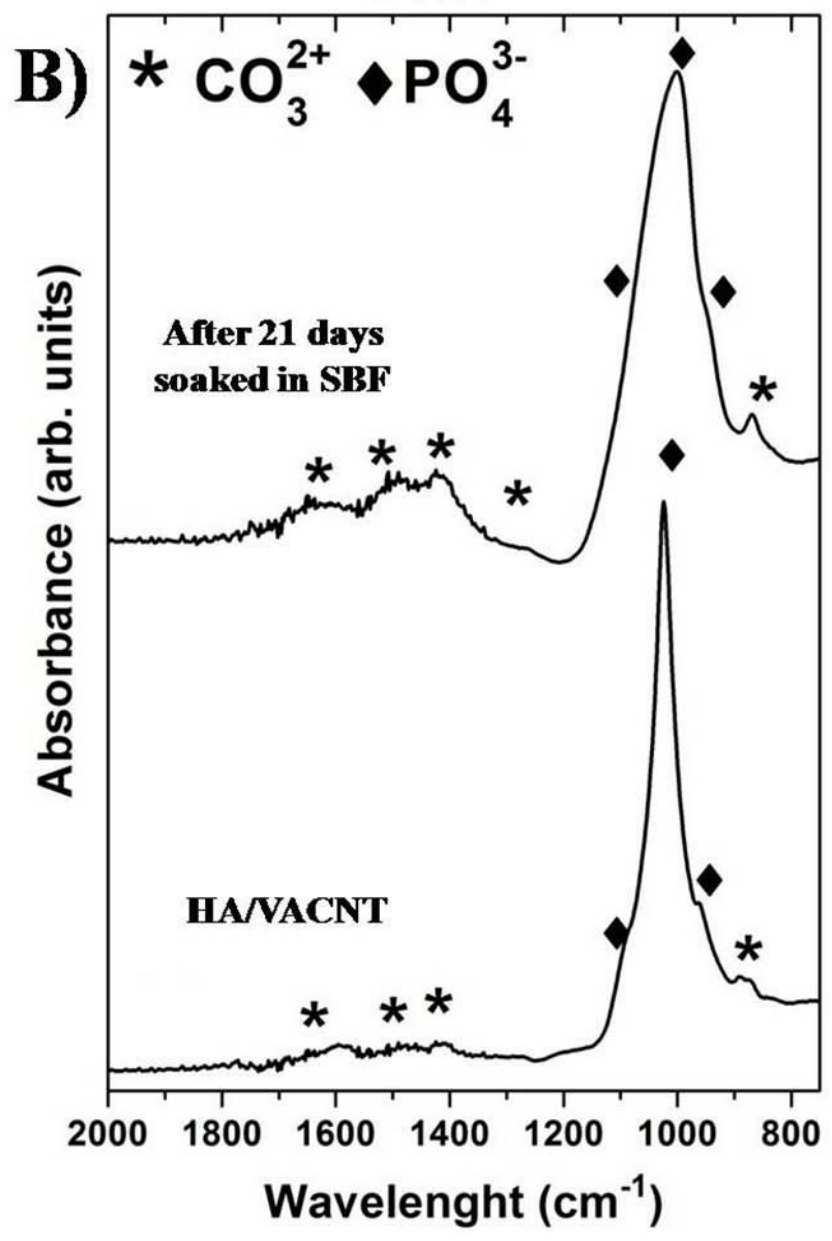

Figure 3. Bioactivity characterization of HA/VACNT nanocomposites using: (A) X-ray diffraction patterns of the superhydrophilic VACNT films, HA/VACNT nanocomposites obtained by electrodeposition technique and after 21 days soaked in SBF. (B) FTIR (ATR) optical technique shows the biological apatites obtained on the HA/VACNT nanocomposites before and after 21 days soaked in SBF.

Figure 4 shows the elemental composition of the coating was investigated by $\mu$ EDX mapping analysis of biological apatites grown on the superhydrophilic HA/VACNTs before (a) and after 21 days (b) soaked in SBF. left pictures are a $\mu \mathrm{EDX}$ line mapping analysis (1) and, the right pictures are shows the quantitative values (mass \%) versus line length media analyses in these different times of soaked in SBF (2). The Ca and P content profiles (\% wt) as a function of depth $(\mu \mathrm{m})$ for each group are shown. The $\mathrm{Ca} / \mathrm{P}$ ratios determined from the analyses were: (a) 1.13 (as-grown superhydrophilic HA/VACNT) and 1.55 (21 days soaked in SBF). These data were collected from the data media of 3 points, area: 0.8 $\mathrm{mm} \times 0.6 \mathrm{~mm}$. All the data shows values near of the biological apatites presented in bone tissue (14-16). It is very 
illustrative and corroborate with the SEM, FTIR and X-Ray analyses to prove the bioactivity of the superhydrophilic HA/VACNT films. Biomimetic bone tissue engineering materials could be used to restore and improve damaged bone. The objective of the study was the generation of HA/VACNT nanocomposites to induce calcification in vitro. Carbon nanotubes appear to be privileged candidates for inducing the mineralization of biological apatites.
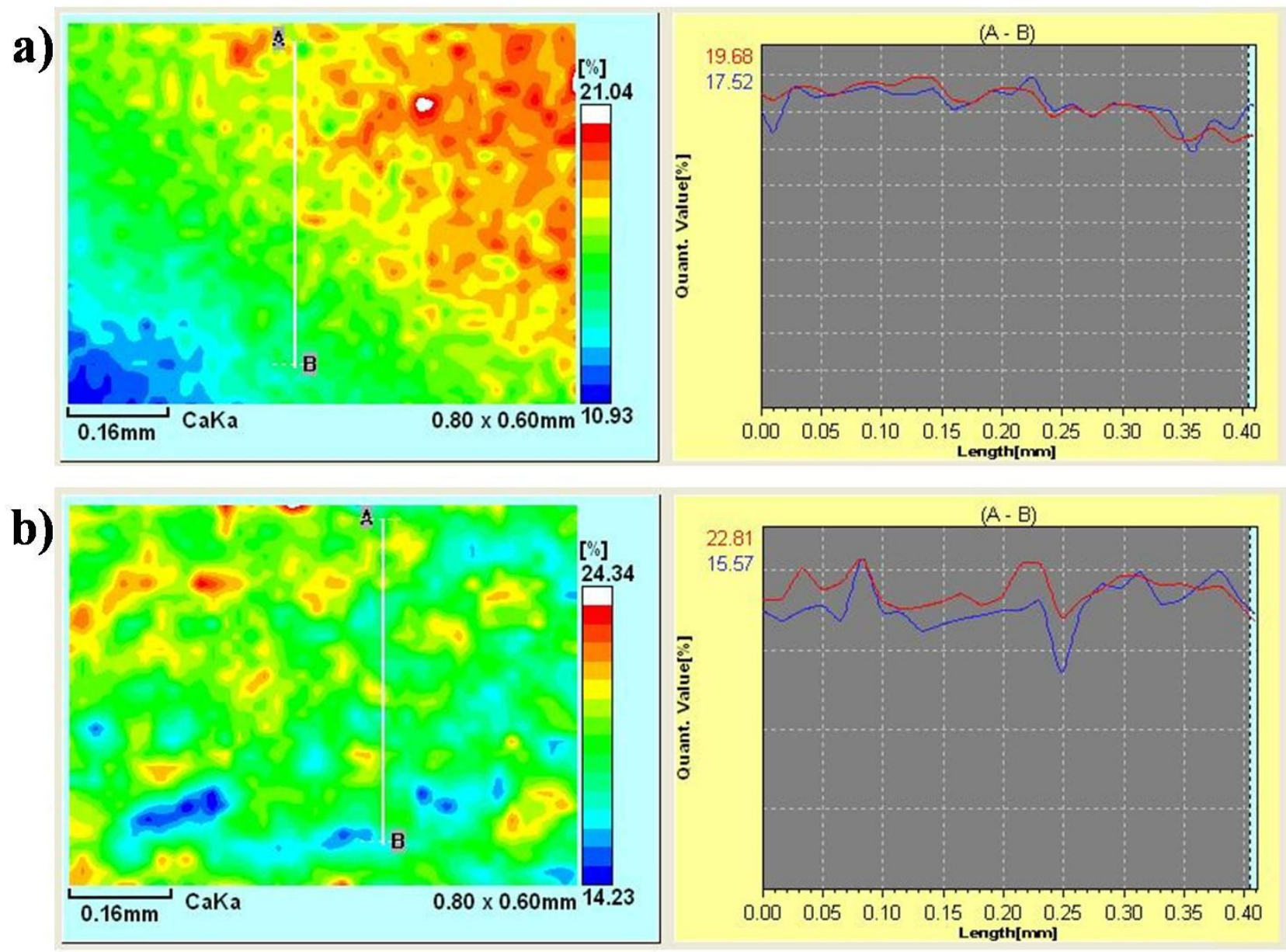

Figure 4. Micro X-ray fluorescence spectrometer by energy-dispersive mapping of biological apatites formed on superhydrophilic VACNT films after (a) 7, (b) 14 and (c) 21 days soaked in simulated body fluid. (1) show the mapping analyses and (2) quantitative values (mass \%) versus line length media.

\section{CONCLUSION}

The results presented here indicate that biomineralization of HA/VACNT scaffolds may become of great interest for biomaterial manufacturing for future use in biomedicine. The superhydrophilic VACNTs films after oxygen plasma treatment presented a new scaffold to direct electrodeposition of HA crystals, and this new nanocomposite shown an excellent bioactivity characteristic that induces the in vitro biomineralization with biological apatites deposition. 


\section{REFERENCES}

[1] Chen, Y., Gan, C., Zhang, T., Yu, G. Appl. Phys Lett. 86, 251905- 251907 (2005) .

[2] Oyane, A., Onuma, K., Ito, A., Kim, H.M., T. Kokubo, Nakamura, T. J. Biomed Mater Res Part A. 64A, 188195 (2003).

[3] Estroff, L.A., Hamilton, A.D. Chem. Mater. 13, 3227-3235, (2001).

[4] Harrison, B.S., Atala, A. Biomaterials 28, 344-353 (2007).

[5] Peppas, N.A., Langer, R. Science. 263, 1715-1720 (1994).

[6] Endo, M.; Strano, M.S.; Ajayan, P.M. Top. Appl. Phys. 111, 13-61 (2008).

[7] Lobo, A.O., Corat, M.A.F., Antunes, E.F., Palma, M.B.S., Pacheco-Soares, C., Garcia, E.E., Corat, E.J. Carbon. 48, 245-254 (2010).

[8] Ramos, S.C., Vasconcelos, G.; E.F. Antunes, A.O. Lobo, V.J. Trava-Airoldi, E.J. Corat, J. Vac Sci Techn B. 28 (2010) 1153-1157.

[9] A.O. Lobo, S.C. Ramos, E.F. Antunes, F.R. Marciano, V.J. Trava-Airoldi, E.J. Corat, J. Mat. Letters. 70 (2011) 89-97.

[10] A.O. Lobo, M.A.F. Corat, S.C. Ramos, J.T. Matsushima, A.E.C. Granato, Langmuir. 26 (2010) 18308-18314.

[11] F. Barrere, M.M.E. Snel, A. Blitterswijka, G. Klaas, P. Layrolle, Biomaterials. 25 (2004) 2901-2910.

[12] Soares, L.E.S.; Brugnera Junior, A.; Zanin, F.A.A.; Santo, A.M.E.; Martin, A.A. J. of Biom. Opt. 2009, 14, n2 024002-1-024002-7.

[13] J.C. Elliott, Recent Studies of Apatites and other Calcium Orthophospates. Calcium Phosphate Materials, Fundamentals, Bres E., Hardouin P., Eds.; Sauramps Medical: Monpellier, 1998, p. 25-66.

[14] F. Swanson, Natl. Bur. Stand. U.S. 1953 II, 51 Circ. 539.

[15] D. Tasis, S. Pispas, C. Galiots, N. Bouropoulos, Mater. Lett 61 (2007) 5044-5046.

[16] S. Aryal, S.R. Bhattarai, B.K.C. Remant, M.S. Khil, L. Duck-Rae, H.Y. Kim, Mater. Sci Eng A. 426 (2006) 202-207. 